\title{
切割方向对桦木衍生的取向微通道生物质炭 锂硫电池隔膜性能的影响
}

\author{
蒋浩，吴淏，侯成义，李耀刚，肖茹，张青红，王宏志 \\ (东华大学 材料科学与工程学院, 纤维材料改性国家重点实验室, 上海 201620)
}

\begin{abstract}
摘 要: 生物源材料由于来源丰富、可循环使用、无污染, 并且能够实现多功能化而引起了广泛关注。本研究利用 大自然中广泛分布的桦木树干为原料, 通过不同取向切割、去木质素和碳化等过程得到具有相应取向的微孔道结构 的生物质炭, 并用作锂硫电池的隔层。生物质炭的比表面积为 $267.7 \mathrm{~m}^{2} / \mathrm{g}$, 有大量的微孔及介孔。测试结果表明: 沿 与电极平面呈 $45^{\circ}$ 方向切割所得的生物质炭的电化学性能最好。在 $0.2 C(1 C=1650 \mathrm{~mA} / \mathrm{g})$ 下该生物质炭隔层制备的锂 硫电池初始比容量为 $979.4 \mathrm{mAh} / \mathrm{g}, 200$ 次循环后保留有 $625.4 \mathrm{mAh} / \mathrm{g}$, 每圈容量损失率仅为 $0.18 \%$ 。该生物质炭隔 层可以有效地吸附和阻挡多硫化锂, 减小充放电过程中产生的穿梭效应, 并且桦木的微通道结构和类蒸腾特性可 以有效地提高电池的比容量、循环稳定性, 有利于锂硫电池的商业化应用。
\end{abstract}

关 键 词: 锂硫电池; 仿生; 桦木; 隔层

中图分类号: TQ174 文献标识码: A

\section{Sawing Angles on Property of Lithium-sulfur Battery Interlayer Prepared with Birch Derived Orientedly Microchannel Biochar}

\author{
JIANG Hao, WU Hao, HOU Chengyi, LI Yaogang, XIAO Ru, ZHANG Qinghong, WANG Hongzhi
}

(State Key Laboratory for Modification of Chemical Fibers and Polymer Materials, College of Materials Science and Engineering, Donghua University, Shanghai 201620, China)

\begin{abstract}
Bio-source materials have attracted wide attention due to their rich sources, recyclability, pollution-free and multi-functional properties. Herein, a lithium-sulfur battery interlayer was obtained by slicing the birch at different angles, following with the delignification and carbonization processes. Biochar containing lots of micropores and mesopores shows a specific surface area up to $267.7 \mathrm{~m}^{2} / \mathrm{g}$. The biochar interlayer sawing along $45^{\circ}$ achieved the highest electrochemical performances. Based on this biochar interlayer, lithium-sulfur battery exhibits a high initial capacity of $979.4 \mathrm{mAh} / \mathrm{g}$ at $0.2 C(1 C=1650 \mathrm{~mA} / \mathrm{g})$ and retains a discharge specific capacity of $625.4 \mathrm{mAh} / \mathrm{g}$ after 200 cycles, which degrades only $0.18 \%$ per cycle. Furthermore, the hierarchical microchannel and transpiration of birch effectively adsorb polysulfide and reduce the shuttle effect, suggesting that it can be widely employed in commercial lithium sulfur battery.
\end{abstract}

Key words: lithium-sulfur battery; bionics; birch; interlayer

收稿日期: 2019-06-12; 收到修改稿日期：2019-09-02

基金项目：中央高校基本科研业务费专项资金(2232019A3-02); 国家自然科学基金(51672043); 东华大学励志计划(LZB2019002) Fundamental Research Funds for the Central Universities (2232019A3-02); National Natural Science Foundation of China (51672043); DHU Distinguished Young Professor Program (LZB2019002)

作者简介: 蒋 浩(1998-), 男, 本科生. E-mail: jianghao3713@gmail.com JIANG Hao(1998-), male, undergraduate. E-mail: jianghao3713@gmail.com

通讯作者: 王宏志, 教授. E-mail: wanghz@dhu.edu.cn; 张青红, 研究员. E-mail: zhangqh@dhu.edu.cn WANG Hongzhi, professor. E-mail: wanghz@dhu.edu.cn; ZHANG Qinghong, professor. E-mail: zhangqh@dhu.edu.cn 
断提高, 当前迫切需要一种比能量高、能量密度高、 成本低且环境友好的新型电池。锂硫电池因具有理 论容量 $(1675 \mathrm{mAh} / \mathrm{g})$ 高、能量密度 $(2600 \mathrm{Wh} / \mathrm{kg})$ 高、 生态友好、硫元素成本低且自然储量丰富等优点, 而成为研究热点 ${ }^{[1-6]}$ 。然而, 锂硫电池仍存在一些问 题, 最主要的是多硫化物中间体 $\left(\mathrm{Li}_{2} \mathrm{~S}_{x}, 4 \leqslant x \leqslant 8\right)$ 的 穿梭效应 ${ }^{[7-10]}$ 。正极产生的多硫化物中间体易溶于 电解液中, 穿过隔膜, 向负极进行扩散, 从而和负 极的金属锂直接发生反应, 使得锂硫电池的容量、 循环寿命和库仑效率迅速下降, 限制了锂硫电池的 应用 ${ }^{[11]}$ 。

近年来, 为了克服穿梭效应, 提高锂硫电池的 电化学性能, 人们采取了多种改进措施, 如电极结 构设计、电解液体系创新和隔层改性等方法 ${ }^{[12-21]}$ 。 其中, 对隔层进行改性是一种极具发展潜力的方法, 通过改性隔层阻止多硫化物溶解到电解液中, 从而 进一步提高正极中硫的利用率 ${ }^{[22]}$ 。利用高比表面积 的碳材料物理吸附多硫化物和极性氧化物化学吸附 多硫化物是抑制穿梭效应的主要方法。在这类改性 隔层中, 介孔 $\mathrm{MnO}_{2}$ 纤维、纳米 $\mathrm{TiO}_{2} 、 \mathrm{Ti}_{4} \mathrm{O}_{7}$ 和 $\mathrm{SiO}_{2}$ 等非碳改性隔层尽管具有较强的多硫化物吸附能 力 $^{[23-26]}$, 但它们的导电性普遍较低, 不能有效地传 输电子, 导致其循环稳定性较差。多孔碳材料因为 具有高比表面积和高导电性 ${ }^{[27]}$, 而且通过吸附作用 能够有效地抑制多硫化物的扩散, 所以具有很好的 发展潜力。

桦树作为天然植物, 在自然界分布广泛, 并且 树干中分布着平行排列的多级结构导管, 为养料和 水分的输送提供了运输通道。受到这种天然结构的 启发, 结合最新仿生学, 本研究提出了一种以天然 桦木为原料来制备锂硫电池隔层的方法。通过简单 的去木质素和碳化过程, 将木材制备成生物质炭隔 层。由于所得到的隔层中有微通道结构, 其比表面 积较大, 从而提高了电池反应中对多硫化物中间体 的物理吸附作用。

\section{1 实验方法}

\section{1 生物质炭隔层的制备}

取桦树的树干洗净、烘干, 采用台式电锯进行 不同取向的切割, 得到不同取向, 即与水平方向分 别成 $0^{\circ} 、 30^{\circ} 、 45^{\circ} 、 60^{\circ}$ 和 $90^{\circ}$ 的木条, 其长度、 宽度和厚度分别为 $10 \mathrm{~cm} 、 3 \mathrm{~cm}$ 和 $1 \mathrm{~mm}$ 。配制 $\mathrm{NaOH}$ 和 $\mathrm{Na}_{2} \mathrm{SO}_{3}$ 混合液, 其浓度分别为 $10.0 \mathrm{wt} \%$ 和 $5.0 \mathrm{wt} \%$, 将桦木条浸没于混合液并转移至水热反应 釜中, 于 $110{ }^{\circ} \mathrm{C}$ 下反应 $12 \mathrm{~h}$ 。反应完毕后, 用 $60{ }^{\circ} \mathrm{C}$
的乙醇、去离子水依次清洗, 并冷冻干燥 $24 \mathrm{~h}$, 即得 到无木质素的桦木条。将桦木条放入坩埚, 置于管 式炉中, 先通高纯氩气(Ar) $1 \mathrm{~h}$ 除去氧气, 气流速度 为 $200 \mathrm{~mL} / \mathrm{min}$ 。以 $5{ }^{\circ} \mathrm{C} / \mathrm{min}$ 的速度升温, 使炉内温 度升至 $250{ }^{\circ} \mathrm{C}$, 保温 $6 \mathrm{~h}$, 进行预碳化, 再以相同升 温速率使炉内温度升至 $1100{ }^{\circ} \mathrm{C}$, 保温 $6 \mathrm{~h}$ 进行碳化, 碳化结束后, 待炉内温度自然冷却至 $100{ }^{\circ} \mathrm{C}$ 以下, 取出后裁剪成长宽为 $10 \sim 11 \mathrm{~mm}$ 、厚度为 $1 \sim 1.2 \mathrm{~mm}$ 的生物质炭片, 即得到所述的生物质炭隔层。

\section{2 材料表征}

使用日本 Hitachi 公司的 SU-8010 型场发射扫描 电子显微镜 (FESEM) 对生物质炭的形貌进行观 察。采用日本电子株式会社的 JEOL JEM-2100F 场 发射透射电子显微镜对生物质炭进行表征。采用英 国 KRATOS 公司的 AXIS-ULTRA DLD 型 X 射线光 电子能谱仪进行 XPS 表征。采用扫描电子显微镜附 件英国牛津仪器公司 EDS Inca X-Max 型能谱仪进 行元素分布的扫描。采用美国康塔公司的 AutosorbiQ 全自动比表面积和孔径分布分析仪进行比表面 积测定(BET)。采用英国雷尼绍公司的 inVia Reflex 显微拉曼光谱仪对材料进行拉曼测试。采用奥地利 安东帕公司的 SAXSess mc2 X 射线衍射仪测试样品 的物相, 辐射源为 $\mathrm{CuK} \alpha(\lambda=0.15406 \mathrm{~nm})$ 。

\section{3 电池的组装和电化学测试}

将活性炭与升华硫按质量比 1:6 碾碎、充分混 合后, 在 $155{ }^{\circ} \mathrm{C}$ 下加热 $10 \mathrm{~h}$, 自然冷却后得到碳硫 $(\mathrm{C} / \mathrm{S})$ 复合材料, 将 $\mathrm{C} / \mathrm{S}$ 复合材料、乙炔黑与聚偏氟 乙烯(PVDF)按质量比 7:2:1 均匀分散于 $N$-甲基吡 咯烷酮(NMP) 溶剂中, 然后把所得浆体均匀涂覆于 铝䇴上。在 $70{ }^{\circ} \mathrm{C}$ 下真空干燥 $24 \mathrm{~h}$, 裁成直径为 $10 \mathrm{~mm}$ 的电极片。以锂片为负极, 分别以 $0^{\circ} 、 30^{\circ} 、 45^{\circ} 、 60^{\circ}$ 和 $90^{\circ}$ 取向的生物质炭与聚丙烯 $(\mathrm{PP})$ 隔膜复合作为生 物质炭-PP 隔层, 使用 $2 \mathrm{vol} \%$ 硝酸锂和 $1 \mathrm{~mol} / \mathrm{L}$ 二(三 氟甲基磺酸)亚胺锂的 1,3 -二氧戊环与乙二醇二甲醚 $(1 / 1, V / V)$ 混合溶液作为电解液, 在手套箱中组装成 2032 型扣式电池(分别记为电池 $\mathrm{A}-0^{\circ} 、 30^{\circ} 、 45^{\circ} 、 60^{\circ}$ 和 $90^{\circ}$ ), 制备流程如图 1 所示。仅用 PP 隔膜组装的 $\mathrm{Li}-\mathrm{S}$ 电池, 记为电池 $\mathrm{B}$ 。此外, 为探究生物质炭隔层 对电池性能的影响, 仅以生物质炭作为正极经上述 相同步骤组装成生物质炭锂硫电池, 记为电池 $\mathrm{C}$ 。 采用武汉蓝电电池测试系统对上述电池进行恒流充 放电测试, 充放电区间为 1.7 2.6 V。使用 CHI660 电化学工作站(上海, 辰华)进行循环伏安测试, 初 始电压为 $2.6 \mathrm{~V}$, 电压范围为 $1.7 \sim 2.6 \mathrm{~V}$, 扫描速度为 $0.1 \mathrm{mV} / \mathrm{s}$ 。 


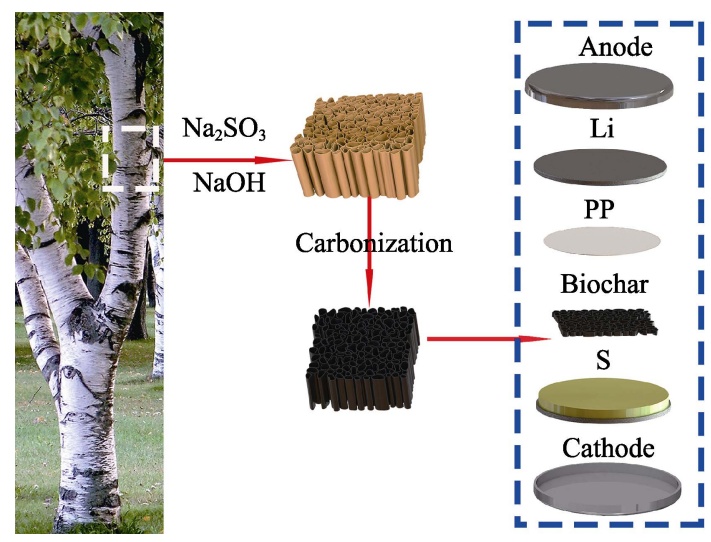

图 1 生物质炭隔层制备示意图

Fig. 1 Schematic diagram of Li-S battery using biochar interlayer

\section{2 结果与讨论}

\section{1 样品的形貌与结构}

图 2(a e)为不同取向生物质炭隔层的截面 SEM 照片。从图中可以看出各个生物质炭隔层的取向大 体符合 $0^{\circ} 、 30^{\circ} 、 45^{\circ} 、 60^{\circ}$ 和 $90^{\circ}$, 其中细微差别主 要是在去木质素和碳化过程中材料发生的不可避免 的微小形变所导致的。插图为各个取向的表面形貌 照片。图 2(f)为沿 $45^{\circ}$ 方向切割的去除木质素前后桦 木片微通道结构的 SEM 照片, 可看出已除木质素 的桦木的导管呈现疏松多孔结构, 而未除的导管内 充满木质素(插图)。疏松多孔的华木能够提供更多 平行排列的多级微通道结构, 使其能够具有更高 的比表面积，在抑制多硫化锂扩散上有更加优异的 表现。

图 $2(\mathrm{~g} \sim \mathrm{h})$ 为 $45^{\circ}$ 取向的生物质炭的 TEM 和 HR-TEM 照片, 其中颜色较深的颗粒为生物质炭, 直径约为 $2 \mathrm{~nm}$ 、长度约为 $10 \mathrm{~nm}$, 白色区域为孔隙。 观察到木材纤维素在碳化过程中由于释放了 $\mathrm{H} 、 \mathrm{~N}$ 、 $\mathrm{O}$ 等非碳元素而发生收缩, 在微通道孔壁上形成了 大量的介孔和蠕虫状微孔结构 ${ }^{[28]}$ 。介孔直径在 10 20 nm 之间, 可提供较短的电子和离子传输通 路 ${ }^{[29]}$ 。而且这些介孔使生物质炭具有较大的比表面 积, 为电解质和电极之间提供更大的接触面积。此 外, HR-TEM 照片进一步证实了该孔隙是由无定型 碳一次颗粒聚集所形成的 ${ }^{[30]}$ 。

图 3(a c) 为 $45^{\circ}$ 取向的生物质炭的 SEM 照片和 相应的 C、S 元素分布图。生物质炭的微通道状结 构清晰可见, 其中主要为 $\mathrm{C}$ 元素, 极少量 $\mathrm{S}$ 元素在 生物质炭中分布均匀, 表明制备得到的生物质炭隔 层主要由碳构成。因此, 可证明在锂硫电池充放电 中主要是碳对多硫化物起到吸附作用。

图 3(d f) 分别为 $45^{\circ}$ 取向的生物质炭 XPS 总谱
图、 $\mathrm{C} 1 \mathrm{~s}$ 和 $\mathrm{S} 2 \mathrm{p}$ 的分谱图。从总谱图中可看到该生 物质炭主要由碳元素组成, 此外少量的氧和硫元素 主要是前期在去木质素过程中, $\mathrm{NaOH}$ 和 $\mathrm{Na}_{2} \mathrm{SO}_{3}$ 不 可避免地残留在材料中。图 3(e) C1s 谱图中, 285.0 和 $285.7 \mathrm{eV}$ 结合能处的峰分别对应 $\mathrm{C}-\mathrm{C} / \mathrm{C}=\mathrm{C}$ 和 $\mathrm{C}-\mathrm{S}$ 键，表明碳材料中的 C 和 S 相互成键，增加了碳 材料的极性，从而增强了对多硫化物的吸附作用 ${ }^{[31]}$ 。

图 3(f) S2p 谱图中, 164.1 和 $165.2 \mathrm{eV}$ 处的峰分别 对应于 $S 2 p^{3 / 2}$ 和 $S 2 p^{1 / 2}$ 的电子结合能, 且 162.1 和 $169.4 \mathrm{eV}$ 分别对应 $\mathrm{Na}-\mathrm{S}$ 金属键和高温形成的硫代 硫酸盐 ${ }^{[32-33]}$ 。

比表面积在电化学领域有着重要的作用, 大的 比表面积在反应过程中往往可以提供更多的反应活 性位点。用 BET 测试生物质炭的比表面积, 沿 $45^{\circ}$ 方向切割的未处理桦木片比表面积为 $1.5 \mathrm{~m}^{2} / \mathrm{g}$, 去 木质素后增加至 $3.4 \mathrm{~m}^{2} / \mathrm{g}$, 经碳化后进一步增加至 $267.7 \mathrm{~m}^{2} / \mathrm{g}$ 。结果表明, 碳化处理的过程显著提高了 原材料的比表面积。图 4(a c) 为沿 $45^{\circ}$ 切割未处理的 桦木片、沿 $45^{\circ}$ 切割去木质素后的桦木片和 $45^{\circ}$ 取向 的生物质炭的 $\mathrm{N}_{2}$ 等温吸-脱附曲线。图 4(c) 在相对 压力较低的区域有一个陡峭的上升，对应其中的微 孔, 在相对压力 0.1 到 0.8 有一个相对较宽的回滞, 对应其中分布较宽的晶粒间堆积形成的介孔。结果 显示材料的孔径集中分布在 $0.5 \sim 1.2 \mathrm{~nm}$ ，高比表面 积使得生物质炭隔层具有优异的物理吸附能力，有 望在锂硫电池工作过程中更有效地吸附多聚硫化物 中间体，从而达到提升电池性能的目的。

$45^{\circ}$ 取向的生物质炭隔层的 Raman 光谱图如 图 4(d)所示, 在 1340 和 $1593 \mathrm{~cm}^{-1}$ 处出现了两个碳 材料的特征峰。其中, 在 $1340 \mathrm{~cm}^{-1}$ 附近的峰称为 D 峰，属于类金刚石结构中碳电子的 $\mathrm{A}_{1 \mathrm{~g}}$ 联合振动模 式, 对应于石墨片层的边缘碳、微晶石墨和热解不 完全所形成的无序四面体网络状 $\mathrm{sp}^{3}$ 杂化碳原子, 与材料内部存在的晶格缺陷及碳原子的无序排列相 关; $1593 \mathrm{~cm}^{-1}$ 附近的称为 $\mathrm{G}$ 峰, 是石墨碳 $\mathrm{sp}^{2}$ 电子结 构的 $E_{2 g}$ 联合振动模式的典型杂化特征峰 ${ }^{[34-35]}$ 。一 般用 $\mathrm{D}$ 峰和 $\mathrm{G}$ 峰的高度比值 $R\left(I_{\mathrm{D}} / I_{\mathrm{G}}\right)$ 来比较碳材料 的石墨化程度 ${ }^{[36]}, R$ 越小, 石墨化程度越高 ${ }^{[37]}$ 。经过 计算, 生物质炭隔层的 $R$ 约为 1.08 , 表明该生物质 炭隔层由较多 $\mathrm{sp}^{3}$ 杂化碳组成, 且含有较多碳缺陷 和空位。该种碳缺陷和空位在充放电过程中为多硫 化锂的吸附提供了额外的反应活性位点，提高了多 硫化锂在碳材料上的吸附性能, 进一步促进了硫和 多硫化锂可逆反应的进行 ${ }^{[38]}$, 对于抑制多硫化锂的 穿梭效应起到了十分重要的作用 ${ }^{[39-40]}$ 。

由图 4(e)所示的 XRD 图谱可以看出, 45 ${ }^{\circ}$ 取向的 
生物质炭隔层在 $2 \theta=23^{\circ}$ 和 $44^{\circ}$ 附近有两个宽峰, 分 别归属于石墨的(002)和(100)晶面。

\section{2 循环伏安特性}

通过组装电池进行测试, 来研究电池 $\mathrm{A}-45^{\circ}$ 和电 池 $\mathrm{B}$ 的电化学性能。当扫描速率为 $0.1 \mathrm{mV} / \mathrm{s}$ 、扫描范 围在 1.7 2.6 V 时, 电池 $\mathrm{B}$ 前 3 圈的循环伏安曲线见 图 5(a), 电池 A- $45^{\circ}$ 前 4 圈的循环伏安曲线见图 5(b)。 从图 5(b)中可以观察到正极硫与负极锂的反应是一 个复杂的多步反应 ${ }^{[11]}$, 正向扫描过程中有一个在 $2.4 \mathrm{~V}$ 附近较为尖锐的氧化峰, 负向扫描过程中有 两个分别在 2.3 和 $2.0 \mathrm{~V}$ 附近的还原峰。位于 $2.3 \mathrm{~V}$
附近的还原峰对应单质硫还原到长链多硫化锂 $\left(\mathrm{Li}_{2} \mathrm{~S}_{t}, 4 \leqslant t \leqslant 8, t\right.$ 是硫链上的硫原子数目) 的过程; 位于 $2.0 \mathrm{~V}$ 附近的还原峰对应长链多硫化锂继续还 原为短链多硫离子 $\left(\mathrm{S}_{x}^{2-}, x=1 \sim 4, x\right.$ 是硫链上的硫原子 数目)及硫化锂 $\left(\mathrm{Li}_{2} \mathrm{~S}\right)$ 的过程。而位于 $2.4 \mathrm{~V}$ 处的尖锐 氧化峰则对应多硫离子以及 $\mathrm{Li}_{2} \mathrm{~S}$ 氧化生成单质硫的 转变过程 ${ }^{[42]}$ 。从图 5(b)还可以看到, 还原峰和氧化 峰均随循环次数的增加而略有偏移, 说明电极材料 在首圈循环时具有较强的极化, 并且伴随有 SEI 膜 的生成。后三圈循环伏安曲线基本趋向于重合, 表明 电池容量保持良好, 后续的电化学反应趋于稳定 ${ }^{[43]}$ 。
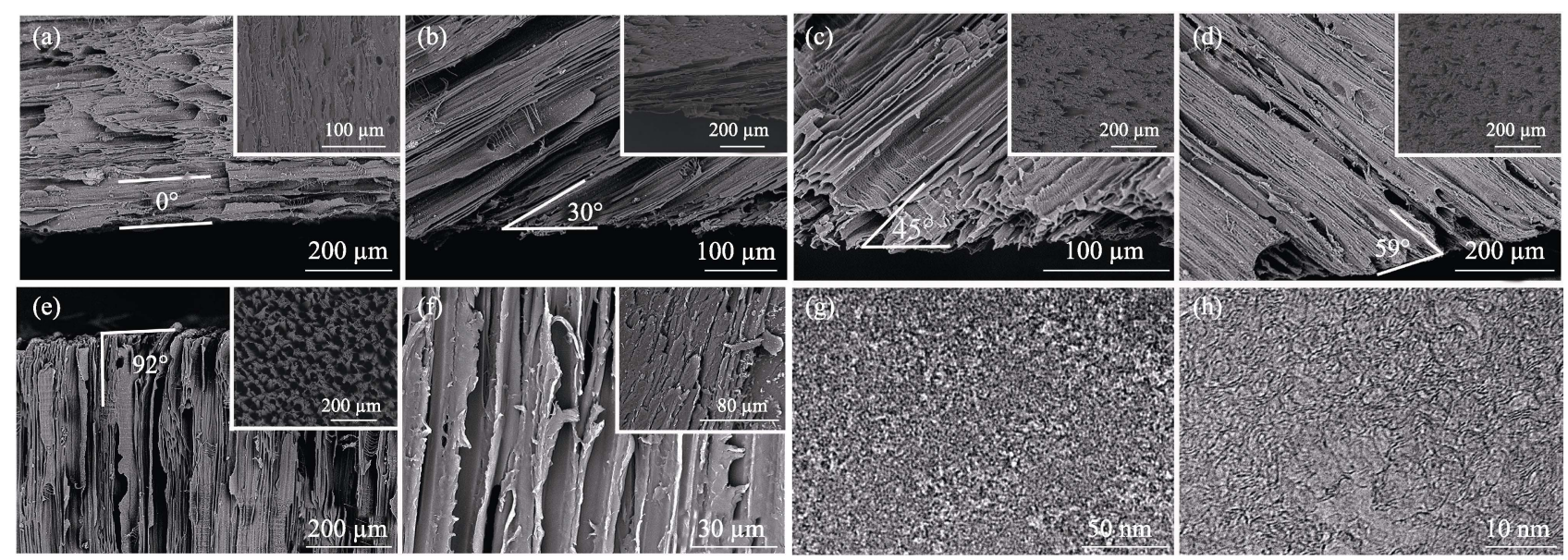

图 2 取向分别为 $0^{\circ}(\mathrm{a}), 30^{\circ}(\mathrm{b}), 45^{\circ}(\mathrm{c}), 60^{\circ}(\mathrm{d})$ 和 $90^{\circ}(\mathrm{e})$ 的生物质炭截面和相应取向(插图)的 SEM 照片; 去除木质素前((f)中插图)后(f)的华木 SEM 照片; 45 取向的生物质炭的 TEM(g)和 HR-TEM(h)照片

Fig. 2 Cross-sectional and longitudinal (inserts) SEM images of the of biochar saw along varying angles: $0^{\circ}(\mathrm{a}), 30^{\circ}(\mathrm{b}), 45^{\circ}(\mathrm{c}), 60^{\circ}(\mathrm{d})$, and $90^{\circ}(\mathrm{e})$; SEM images of wood before (insert in (f)) and after (f) delignification; TEM (g) and HRTEM(h) images of biochar oriented at $45^{\circ}$
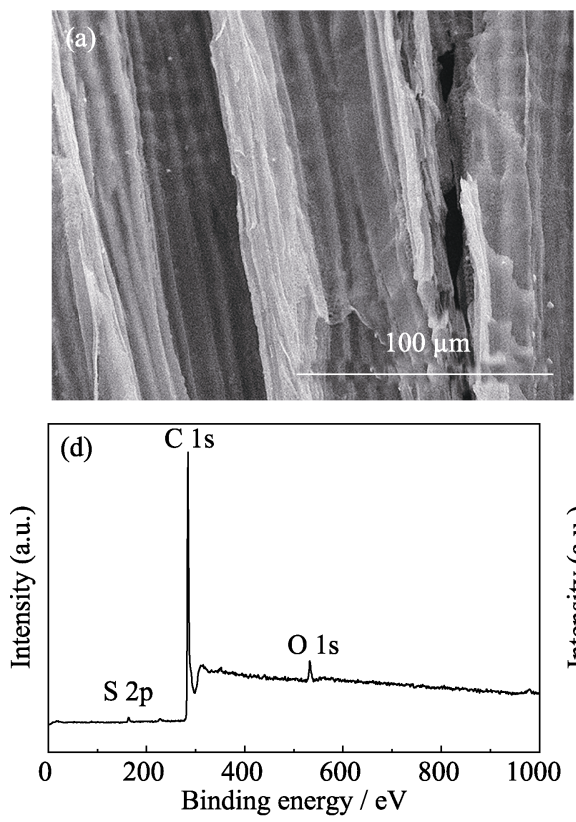
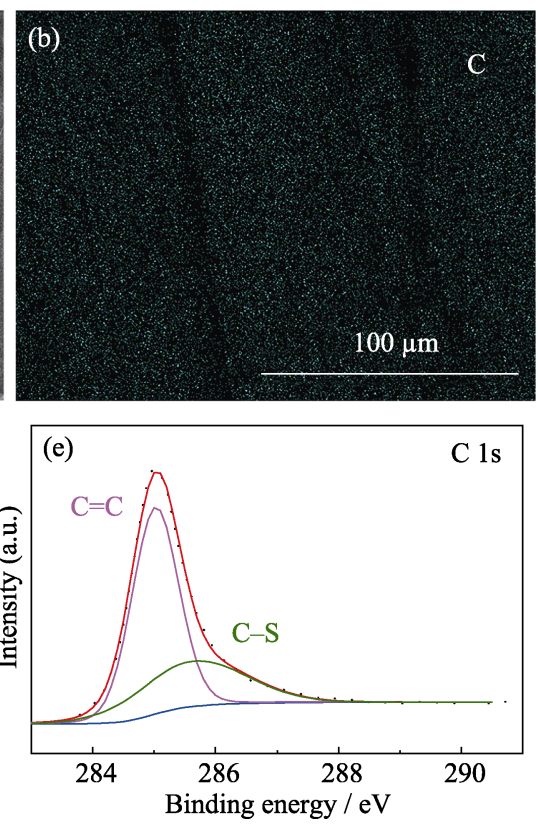
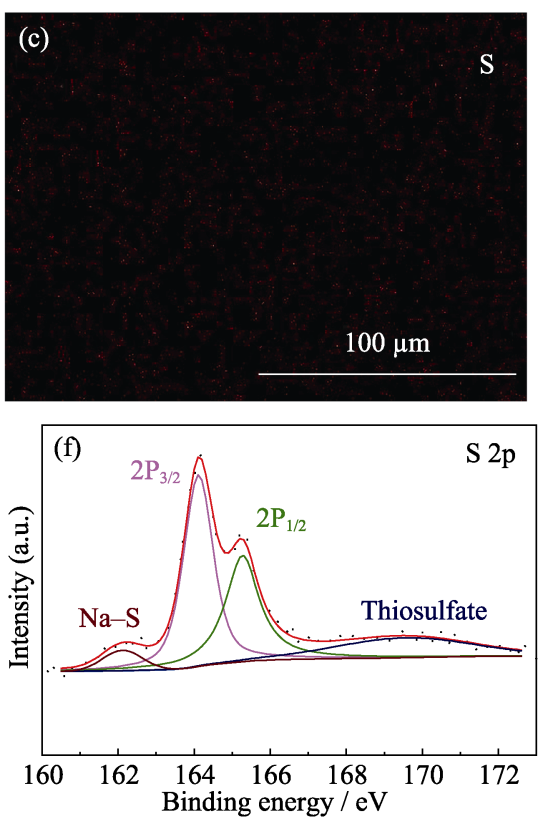

图 $345^{\circ}$ 取向的生物质炭的 SEM 照片(a)以及相应的碳(b)、硫(c)的元素面分布图; (d f) $45^{\circ}$ 取向的生物质炭材料的 XPS 元素分析全谱(d)、C1s (e)和 S2p (f)分谱图

Fig. 3 SEM image (a), corresponding C (b) and S (c) elements distribution mappings of biochar oriented at $45^{\circ}$, XPS spectra of biochar oriented at $45^{\circ}$, survey spectrum (d), C1s (e), and S2p (f) 

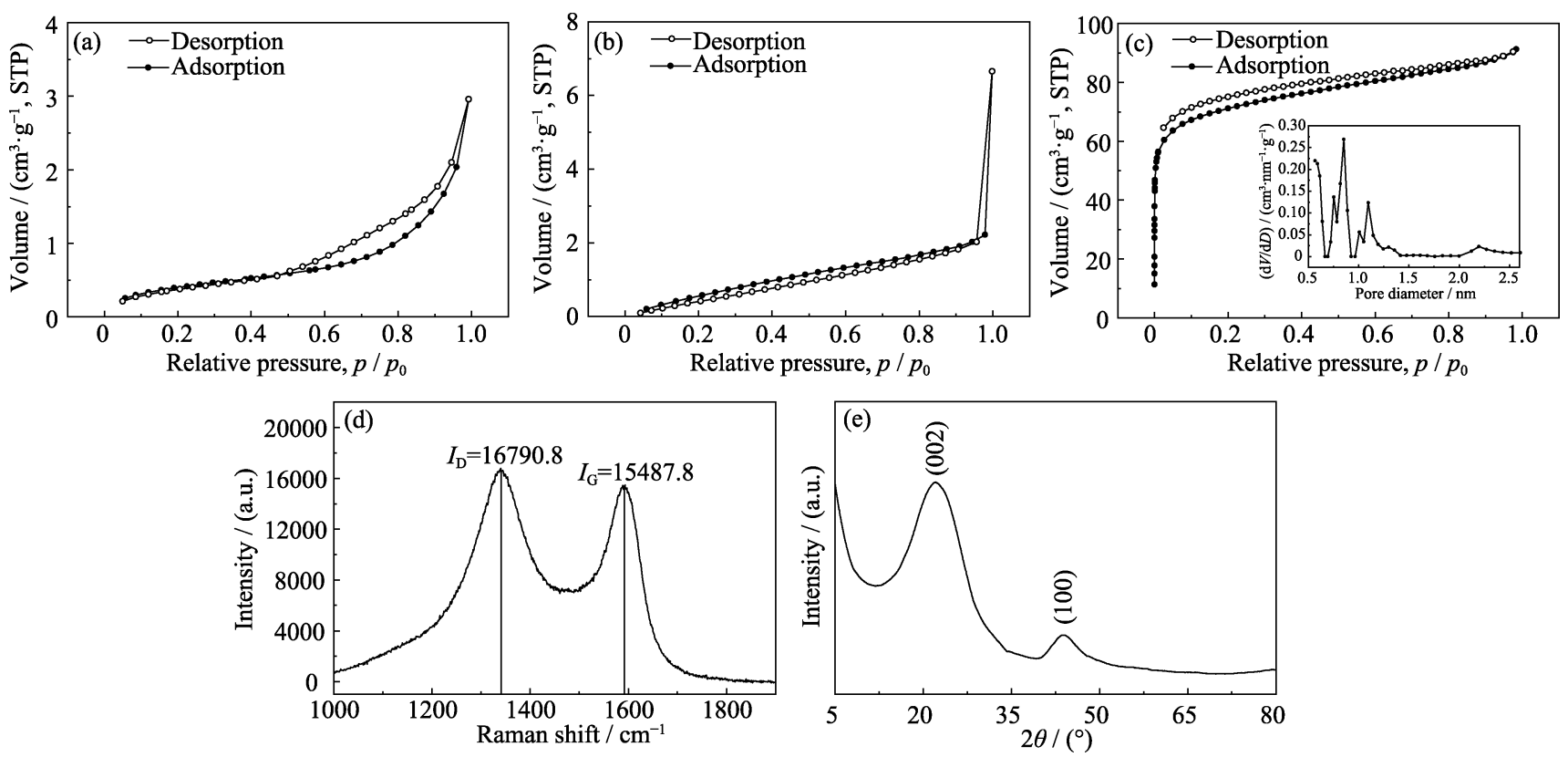

图 $4 \quad(\mathrm{a} \sim \mathrm{c})$ 沿 $45^{\circ}$ 切割所得未处理的桦木片(a)、去木质素后的华木片(b)和 $45^{\circ}$ 取向的生物质炭(c)的 $\mathrm{N}_{2}$ 等温吸-脱附曲线; $45^{\circ}$ 取向生物质炭的孔径分布曲线((c)中插图); (d) $45^{\circ}$ 取向的生物质炭的拉曼光谱图; (e) $45^{\circ}$ 取向的生物质炭 XRD 图谱

Fig. 4 (a-c) $\mathrm{N}_{2}$ adsorption-desorption isotherms of the untreated birch (a), lignin-free birch (b), biochar sawing along $45^{\circ}(\mathrm{c})$ and pore size distribution curve (insert in (c)) of biochar sawing along $45^{\circ}$;

Raman spectrum (d) and XRD pattern (e) of biochar oriented at $45^{\circ}$

可见生物质炭的微通道结构对硫和多硫化物的固定 发挥了重要作用。

\section{3 电池的充放电性能}

为研究电池 $\mathrm{A}$ 的充放电性能, 以及微细导管 不同取向对锂离子扩散和多硫化物扩散的影响, 测试了电池 $\mathrm{A}-0^{\circ} 、 30^{\circ} 、 45^{\circ} 、 60^{\circ}$ 和 $90^{\circ}$ 以及电池 $\mathrm{B}$ 在相同条件下的循环性能, 见图 5(c)。由图可知, 在 $0.2 C$ 的电流密度下, 电池 $\mathrm{A}-45^{\circ}$ 循环性能最好, 且其循环性能较电池 $\mathrm{B}$ 有明显提升, 初始容量为 $979.4 \mathrm{mAh} / \mathrm{g}$ 左右, 经过 200 次循环后放电容量仍 保持在 $625.4 \mathrm{mAh} / \mathrm{g}$, 单次衰减速率约为 $0.18 \%$ 。主 要原因在于生物质炭的物理吸附有效地抑制了电极 反应过程中所产生的多硫化锂中间体的穿梭效应, 从而显著提升了正极硫活性材料的利用率，提高了 锂硫电池的循环性能。同时桦木自身微观多级导管 和宏观 $45^{\circ}$ 取向的结构, 有利于充放电过程中电解 液的浸润。该取向角度有助于阻隔多硫化物向负极 扩散，同时又减小了锂离子的扩散阻力，而且也为 体积膨胀提供了很好的缓冲空间, 防止了结构塌 陷。为探究 $45^{\circ}$ 取向的生物质炭在锂硫电池充放电 过程中对比容量的影响, 以该生物质炭作为正极 组装电池(电池 $\mathrm{C}$ )进行测试, 测试结果见图 5 (c) 插 图, $45^{\circ}$ 取向的电池 $\mathrm{C}$ 首四圈放电比容量分别为 1747.7、218.0、150.3 和 $151.2 \mathrm{mAh} / \mathrm{g}$, 随后比容量 几乎为 0 , 说明生物质炭仅在前四圈提供部分比容
量。这是桦木本身多孔结构使得锂离子在微通道内 富集所导致的。

图 5(d) 为电池 $\mathrm{A}-45^{\circ}$ 在 $0.05 C$ 前 3 圈的充放电曲 线。图中在电压为 $2.3 、 2.1 \mathrm{~V}$ 时有两个放电平台, 电 压为 $2.3 \mathrm{~V}$ 时有一个充电平台, 与图 5(b) 的循环伏安 曲线结果相一致。在 $0.2 C$ 下, 首三圈放电比容量分 别为 3352.3、1936.1 和 $1784.9 \mathrm{mAh} / \mathrm{g}$, 均超出硫的 理论容量, 这主要是生物质炭在前四圈中贡献部分 容量所致。第一个放电平台主要对应单质硫转变为 长链聚硫化锂 $\left(\mathrm{Li}_{2} \mathrm{~S}_{t}, 4 \leqslant t \leqslant 8\right)$ 的还原过程, 第二个 放电平台主要为长链多硫化锂继续还原为短链多硫 离子 $\left(\mathrm{S}_{x}^{2-}, x=1 \sim 4\right)$ 及硫化锂 $\left(\mathrm{Li}_{2} \mathrm{~S}\right)$ 的过程 ${ }^{[44]}$ 。在充电曲 线中, 平台对应硫化锂和多硫离子转变为单质硫的 氧化反应。反应方程式如下 ${ }^{[45]}$ :

放电:

$$
\begin{gathered}
\mathrm{S}_{8}+2 \mathrm{e}^{-} \rightarrow \mathrm{S}_{t}^{2-}(4 \leqslant t \leqslant 8) 2.3 \mathrm{~V} \\
\mathrm{~S}_{t}^{2-}+2 \mathrm{e}^{-} \rightarrow 2 \mathrm{~S}_{x}^{2-}(1 \leqslant x \leqslant 4) 2.1 \mathrm{~V}
\end{gathered}
$$

充电:

$$
8 \mathrm{Li}_{2} \mathrm{~S} \rightarrow 16 \mathrm{e}^{-}+16 \mathrm{Li}^{+}+\mathrm{S}_{8} 2.3 \mathrm{~V}
$$

电池 $\mathrm{A}$ 的容量较高, 一方面是由于生物质炭使 正极的导电性得到了有效提高, 而且作为第二集流 体在充放电过程中有效地促进了单质硫、多硫化锂 和硫化锂的可逆反应 ${ }^{[6]}$ 。另一方面，该隔层利用桦 木本身多级通道结构起到了阻隔和吸附多硫化物的 作用, 使多硫化锂中间体沉积在隔层上, 在其充电 

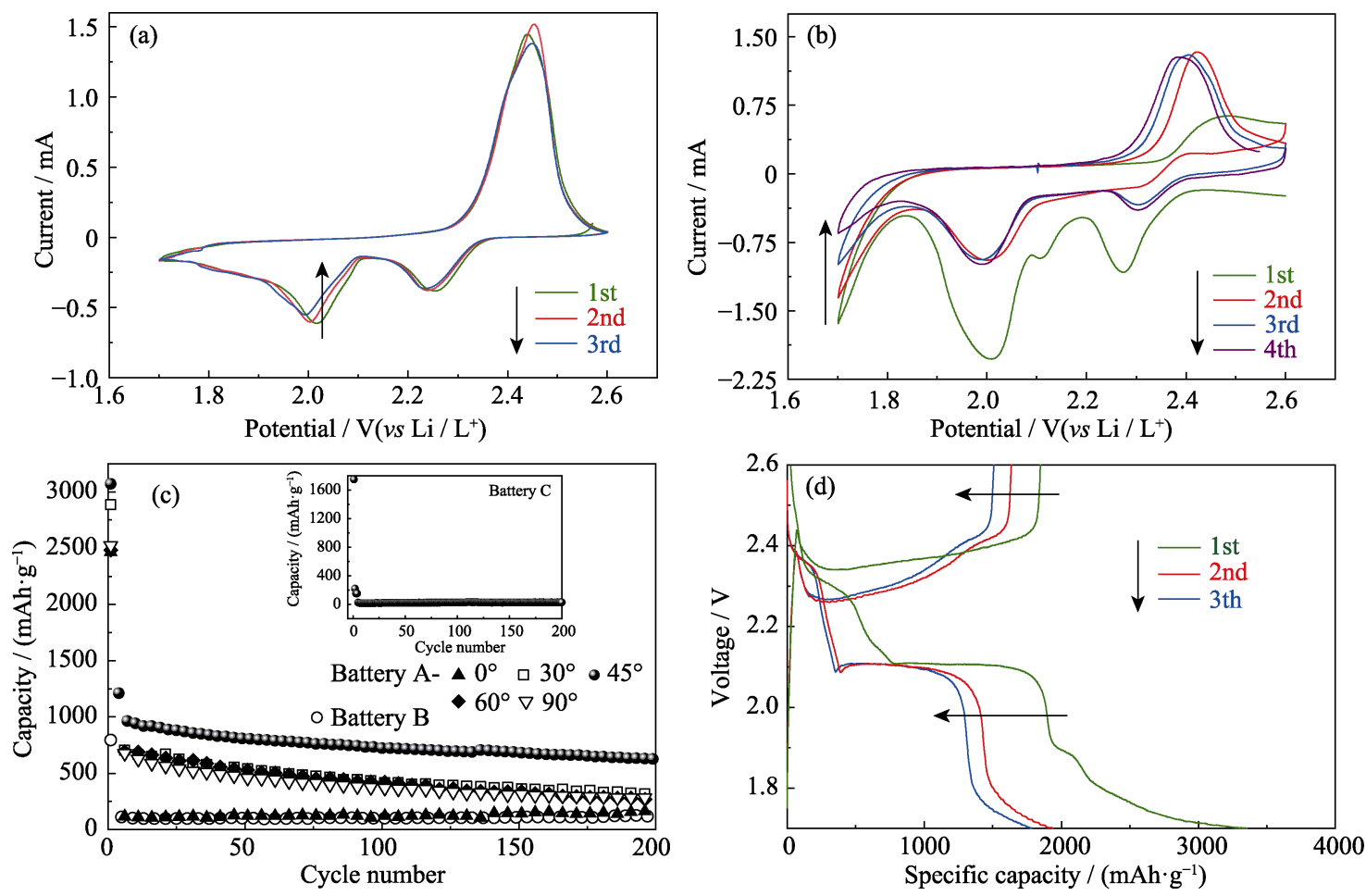

图 5 电池 $\mathrm{B}(\mathrm{a})$ 与电池 $\mathrm{A}-45^{\circ}(\mathrm{b})$ 在电压窗口为 $1.7 \sim 2.6 \mathrm{~V} 、 0.1 \mathrm{mV} / \mathrm{s}$ 时的循环伏安曲线; (c)不同取向的电池 $\mathrm{A}$ 和 电池 $\mathrm{B}$ 循环性能对比图, 插图为电池 $\mathrm{C}$ 循环性能图; (d) $0.05 \mathrm{C}$ 时电池 $\mathrm{A}-45^{\circ}$ 的前三次充放电曲线

Fig. 5 Cyclic voltammograms of battery B (a) and battery A- $45^{\circ}$ (b) between $1.7-2.6 \mathrm{~V}$ at $0.1 \mathrm{mV} / \mathrm{s}$; (c) Cycling performances of batteries A with different cutting orientation biochar interlayers and battery $\mathrm{B}$ with insert showing the cycling performance of battery $\mathrm{C}$; (d) Charging-discharging profiles of battery A- $45^{\circ}$ at $0.05 \mathrm{C}$

时能快速被氧化并重复利用, 提高了正极活性单质 硫的利用率。

\section{3 结论}

1) 利用桦木为原料制备了一种生物质炭改性隔 层, 该隔层能够有效地提高锂硫电池的电化学性能。

2) 利用桦木本身平行排列的微通道结构和高比 表面积 $\left(267.7 \mathrm{~m}^{2} / \mathrm{g}\right)$, 能够在锂硫电池中起到物理阻 隔和吸附多硫化锂的作用，有效地抑制穿梭效应， 减少活性物质损失。

3) 该生物质炭隔层在锂硫电池中不仅可以降低 电极与隔膜之间的电阻，还能起到第二集流体的作 用，进而提高活性物质的利用率和电池比容量。

\section{参考文献:}

[1] ARMAND M, TARASCON J M. Building better batteries. Nature, 2008, 451(7179): 652-657.

[2] BRUCE P G, FREUNBERGER S A, HARDWICK L J, et al. $\mathrm{LiO}_{2}$ and Li-S batteries with high energy storage. Nature Materials, 2012, 11(1): 19-29.

[3] SEH Z W, SUN Y M, ZHANG Q F, et al. Designing high-energy lithium-sulfur batteries. Chemical Society Reviews, 2016, 45(20): 5605-5634.

[4] FANG R P, ZHAO S Y, SUN Z H, et al. More reliable lithiumsulfur batteries: status, solutions and prospects. Advanced Materi- als, 2017, 29(48): 1606823-1-25.

[5] JI X L, LEE K T, NAZAR LINDA F. A highly ordered nanostructured carbon-sulphur cathode for lithium-sulphur batteries. Nature Materials, 2009, 8(6): 500-506.

[6] MANTHIRAM A, CHUNG S H, ZU C X. Lithium-sulfur batteries: progress and prospects. Advanced Materials, 2015, 27(12): 1980-2006.

[7] CHENG X B, YAN C, CHEN X, et al. Implantable solid electrolyte interphase in lithium-metal batteries. Chem., 2017, 2(2): 258-270.

[8] YE H, XIN S, YIN Y X, et al. Advanced porous carbon materials for high-efficient lithium metal anodes. Advanced Energy Materials, 2017, 7(23): 1700530-1-9.

[9] CHEN J J, YUAN R M, FENG J M, et al. Conductive Lewis base matrix to recover the missing link of $\mathrm{Li}_{2} \mathrm{~S}_{8}$ during the sulfur redox cycle in Li-S battery. Chemistry of Materials, 2015, 27(6): 2048-2055.

[10] YU S, FENG X R, ZHANG N, et al. Understanding conversiontype electrodes for lithium rechargeable batteries. Accounts of Chemical Research, 2018, 51(2): 273-281.

[11] EVERS S, NAZAR L F. New approaches for high energy density lithium-sulfur battery cathodes. Accounts of Chemical Research, 2013, 46(5): 1135-1143.

[12] JI L W, RAO M M, ZHENG H M, et al. Graphene oxide as a sulfur immobilizer in high performance lithium/sulfur cells. Journal of The American Chemical Society, 2011, 133(46): 18522-18525.

[13] QIN F R, WANG X W, ZHANG K, et al. High areal capacity cathode and electrolyte reservoir render practical Li-S batteries. Nano Energy, 2017, 38: 137-146.

[14] FU Y Z, SU Y S, MANTHIRAM A. Highly reversible lithium/ dissolved polysulfide batteries with carbon nanotube electrodes. Angewandte Chemie-International Edition, 2013, 52(27): 6930-6935.

[15] CHUNG S H, HAN P, SINGHAL R, et al. Electrochemically stable rechargeable lithium-sulfur batteries with a microporous carbon 
nanofiber filter for polysulfide. Advanced Energy Materials, 2015, 5(18): $1500738-1-11$.

[16] LI Z, WU H B, LOU X W. Rational designs and engineering of hollow micro-/nanostructures as sulfur hosts for advanced lithiumsulfur batteries. Energy \& Environmental Science, 2016, 9(10): 3061-3070.

[17] LUO S W, YAO M J, LEI S, et al. Freestanding reduced graphene oxide-sulfur composite films for highly stable lithium-sulfur batteries. Nanoscale, 2017, 9(14): 4646-4651.

[18] WANG H L, YANG Y, LIANG Y Y, et al. Graphene-wrapped sulfur particles as a rechargeable lithium-sulfur battery cathode material with high capacity and cycling stability. Nano Letters, 2011, 11(7): 2644-2647.

[19] CAO J, CHEN C, ZHAO Q, et al. A flexible nanostructured paper of a reduced graphene oxide-sulfur composite for high-performance lithium-sulfur batteries with unconventional configurations. $A d$ vanced Materials, 2016, 28(43): 9629-9636.

[20] JEONG Y C, KIM J H, NAM S, et al. Rational design of nanostructured functional interlayer/separator for advanced Li-S batteries. Advanced Functional Materials, 2018, 28(38): 1707411-1-32.

[21] WANG P, ZHANG Z A, HONG B, et al. Multifunctional porous $\mathrm{VN}$ nanowires interlayer as polysulfides barrier for high performance lithium sulfur batteries. Journal of Electroanalytical Chemistry, 2019, 832: 475-479.

[22] XU G Y, YAN Q B, WANG S T, et al. A thin multifunctional coating on a separator improves the cyclability and safety of lithium sulfur batteries. Chemical Science, 2017, 8(9): 6619-6625.

[23] LAI Y Q, WANG P, QIN F R, et al. A carbon nanofiber@mesoporous $\delta-\mathrm{MnO}_{2}$ nanosheet-coated separator for high-performance lithiumsulfur batteries. Energy Storage Materials, 2017, 9: 179-187.

[24] PARK J, KIM E T, KIM C, et al. The importance of confined sulfur nanodomains and adjoining electron conductive pathways in subreaction regimes of Li-S batteries. Advanced Energy Materials, 2017, 7(19): 1700074-1-10.

[25] LIANG G M, WU J X, QIN X Y, et al. Ultrafine $\mathrm{TiO}_{2}$ decorated carbon nanofibers as multifunctional interlayer for high-performance lithium-sulfur battery. ACS Applied Materials \& Interfaces, 2016, 8(35): 23105-23113.

[26] REHMAN S, GUO S J, HOU Y L. Rational design of $\mathrm{Si} / \mathrm{SiO}_{2} @$ hierarchical porous carbon spheres as efficient polysulfide reservoirs for high-performance Li-S battery. Advanced Materials, 2016, 28(16): 3167-3172.

[27] HUANG J Q, ZHANG Q, WEI F. Multi-functional separator/ interlayer system for high-stable lithium-sulfur batteries: progress and prospects. Energy Storage Materials, 2015, 1: 127-145.

[28] LIU M C, KONG L B, ZHANG P, et al. Porous wood carbon monolith for high-performance supercapacitors. Electrochimica Acta, 2012, 60: 443-448.

[29] ZHANG S, WU C L, WU W, et al. High performance flexible supercapacitors based on porous wood carbon slices derived from Chinese fir wood scraps. Journal of Power Sources, 2019, 424: 1-7.

[30] MA F W, SONG S J, WU G, et al. Facile selt-template large scale preparation of biomass-derived 3D hierarchical porous carbon for advanced supercapacitors. J. Mater. Chem. A, 2015, 3(35):
18154-18162.

[31] YUAN X Q, WU L S, HE X L, et al. Separator modified with N, S co-doped mesoporous carbon using egg shell as template for high performance lithium-sulfur batteries. Chemical Engineering Journal, 2017, 320: 178-188.

[32] ROSENMAN A, MARKEVICH E, SALITRA G, et al. Facile synthesis and very stable cycling of polyvinylidene dichloride derived carbon: sulfur composite cathode. Journal of The Electrochemical Society, 2016, 163(9): A1829-A1835.

[33] LIANG X, HART C, PANG Q, et al. A highly efficient polysulfide mediator for lithium-sulfur batteries. Nature Communications, 2015, 6: 5682-1-8.

[34] MALARD L M, PIMENTA M A, DRESSELHAUS G, et al. Raman spectroscopy in graphene. Physics Reports, 2009, 473(5): 51-87.

[35] WALlE M D, ZHANG Z, ZHANG M, et al. Hierarchical 3D nitrogen and phosphorous codoped graphene/carbon nanotubessulfur composite with synergistic effect for high performance of lithium-sulfur batteries. Journal of Materials Science, 2018, 53(4): 2685-2696.

[36] FERRARI A, ROBERTSON J. Interpretation of Raman spectra of disordered and amorphous carbon. Physical Review B (Condensed Matter), 2000, 61(20): 14095-14107.

[37] YANG S J, KIM T, IM J H, et al. MOF-derived hierarchically porous carbon with exceptional porosity and hydrogen storage capacity. Chemistry of Materials, 2012, 24(3): 464-470.

[38] OAKES L, CARTER R, PINT C L. Nanoscale defect engineering of lithium-sulfur battery composite cathodes for improved performance. Nanoscale, 2016, 8(46): 19368-19375.

[39] ZHU Z Q, CHENG F Y, CHEN J. Investigation of effects of carbon coating on the electrochemical performance of $\mathrm{Li}_{4} \mathrm{Ti}_{5} \mathrm{O}_{12} / \mathrm{C}$ nanocomposites. Journal of Materials Chemistry A, 2013, 1(33): 9484-9490.

[40] LI Z, ZHANG J T, GUAN B Y, et al. A sulfur host based on titanium monoxide@carbon hollow spheres for advanced lithiumsulfur batteries. Nature Communications, 2016, 7: 13065-1-11.

[41] HUANG J Q, LIU X F, ZHANG Q, et al. Entrapment of sulfur in hierarchical porous graphene for lithium-sulfur batteries with high rate performance from -40 to $60{ }^{\circ} \mathrm{C}$. Nano Energy, 2013, 2(2): 314-321.

[42] FU X, YANG R, LIU L, et al. Preparation of cellular 3-D graphite and its application in Li-S battery. New Chemical Materials, 2019, 47(2): 85-89.

[43] YANG S Y, YAN C, CAO Z X, et al. Preparation of hierarchical porous carbon/sulfur composite based on lotus-leaves and its property for Li-S batteries. Journal of Inorganic Materials, 2016, 31(2): $135-140$.

[44] ZHU J D, GE Y Q, KIM D, et al. A novel separator coated by carbon for achieving exceptional high performance lithium-sulfur batteries. Nano Energy, 2016, 20: 176-184.

[45] FANG X, PENG H S. Revolution in electrodes: recent progress in rechargeable lithium-sulfur batteries. Small, 2015, 11(13): 1488-1511.

[46] SONG J, SU D, XIE X, et al. Immobilizing polysulfides with MXene-functionalized separators for stable lithium-sulfur batteries. ACS Appl. Mater. Interfaces, 2016, 8(43): 29427-29433. 